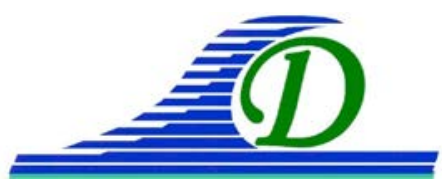

XIII İmes Journées Nationales Génie Côtier - Génie Civil

Dunkerque, 2-4 juillet 2014

DOI:10.5150/jngcgc.2014.012 @ Editions Paralia CFL

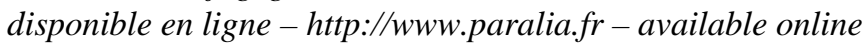

\title{
Développements récents dans la connaissance de l’océanologie du lagon de Wallis
}

\section{Thierry HOIBIAN ${ }^{1}$, Enelio LIUFAU ${ }^{2}$, Michel ALLENBACH ${ }^{1}$, Carole MANRY ${ }^{2}$, Atoloto MALAU ${ }^{2}$}

1. Université de la Nouvelle-Caléonie, PPME - EA3325, BP R4, 98851 Nouméa Cedex, Nouvelle-Calédonie. thierry.hoibian@univ-nc.nc

2. Service Territorial de l'Environnement, BP 294 - Mata'Utu - Uvea, 98600 Wallis et Futuna.

sig.env@mail.wf

Résumé : De nouvelles acquisitions de données courantologiques obtenues dans le suivi océanologique du lagon de Wallis entrepris depuis 2006, ont permis de mieux définir le schéma de circulation général du lagon de Wallis. L’importance des entrées d'eau océaniques dues au déferlement des vagues par-dessus la barrière récifale, mesurées dans plusieurs situations météo-océanologiques, aboutissent à la définition du concept de bourrelet de surpression créé par le déferlement des vagues par-dessus la barrière récifale qui se développe du coté au vent de l'île. L'importance de ce bourrelet de surpression conditionne la circulation dans le lagon par le biais des échanges entre bassins lagonaires au travers des chenaux internes et des passes. En fonction des conditions météo-océanologiques, il se développe une circulation circulaire conduisant au déplacement des masses d'eau excédentaires dans les bassins situés du côté opposé de l'île, et à leur évacuation par les passes et par-dessus le récif.

Mots-clés : Courantologie doppler, Lagon, Marées, Houles, Suivi du réchauffement climatique, Wallis, Pacifique Sud-Ouest.

Abstract: New ADCP current meter acquisitions data recorded in the oceanologic monitoring of the Wallis lagoon, undertaken since 2006, in the context of assessing the impact of global warming, have helped to define a general circulation pattern for the Wallis lagoon. The importance of ocean water inputs due to the waves breaking over the barrier reef, measured in several ocean-weather situations, lead to the definition of a pressure gradient that develops on the windward side of the island. The importance of this pressure gradient affects the residual circulation in the lagoon and the inter basins exchanges through the lagoon channels. This induces a circular movement of the water masses in excess, toward the lagoon basins located on the opposite side of the island, for their evacuation through the passes and over the barrier reef.

Keywords: ADCP current Profiler, Lagoon, Tides, Swells, Global warming, Wallis islands, Southwest Pacific. 


\section{Thème 1 - Hydrodynamique côtière}

\section{Introduction}

Une étude océanologique du lagon de Wallis est en cours de réalisation sur divers financements dont celui de l'IFRECOR. Positionnée par $13^{\circ} 16^{\prime} \mathrm{S}$ et $176^{\circ} 15^{\prime} \mathrm{W}$, l'archipel des îles Wallis est un territoire français d'outre-mer situé dans le Pacifique sud-ouest. L'île principale, est longue de $15 \mathrm{~km}$ et de large de $6 \mathrm{~km}$. Elle est entourée par un récif barrière qui s’étire sur $24 \mathrm{~km}$ dans la direction nord-sud et sur $15 \mathrm{~km}$ dans une direction est-ouest. Le récif délimite un lagon d'une largeur de 3 à $5 \mathrm{~km}$, d'une profondeur moyenne d'environ $20 \mathrm{~m}$ avec des fosses où elle dépasse $60 \mathrm{~m}$. Ce lagon est ouvert sur l'océan au niveau de quatre passes (figure 1).
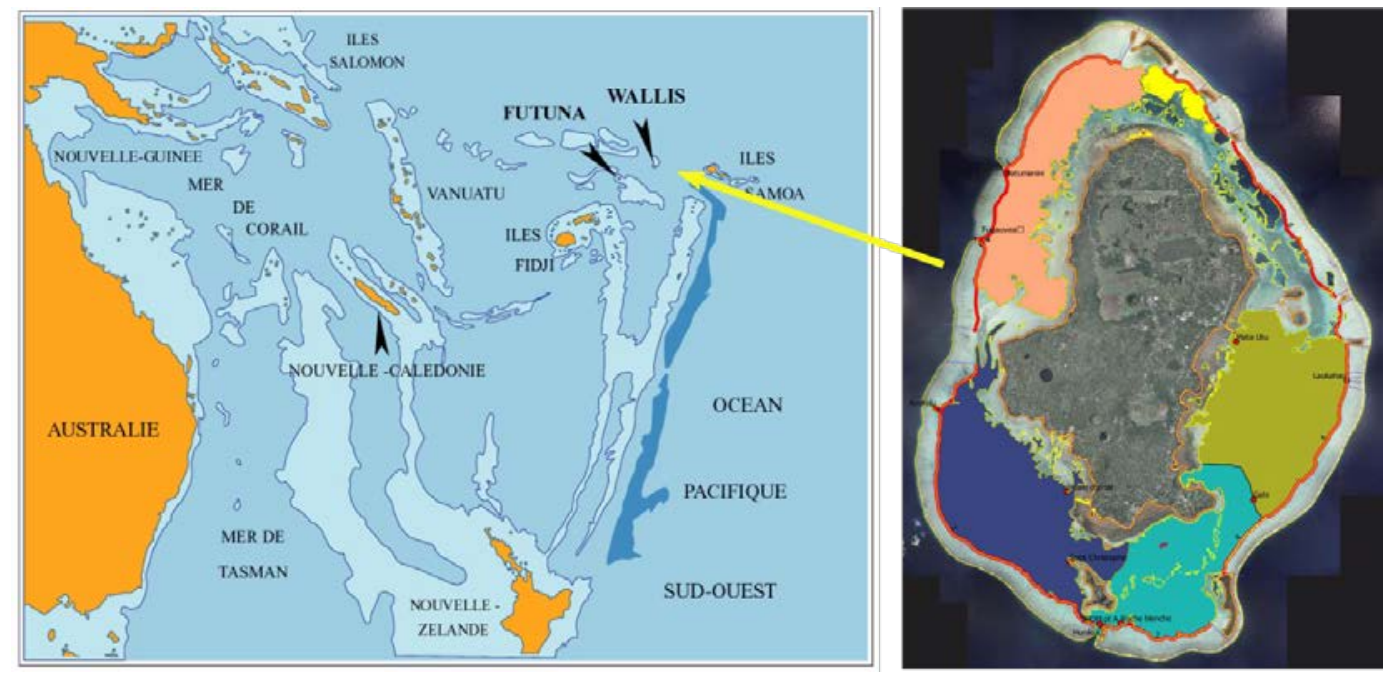

Figure 1. Localisation des îles Wallis dans le Pacifique sud-ouest et délimitation des bassins lagonaires.

Le lagon de Wallis se compose de trois bassins principaux. Le bassin de Est (de Mata Utu ssl.) occupe toute la façade Est de l'île, du nord au sud. La barrière récifale à ce niveau est légèrement surélevée par rapport à la barrière du coté-ouest qui est surbaissée. Elle ne possède pas d'ouverture vers le large et communique avec les autres bassins par des passes internes ; la fausse passe de Vailala au Nord, les passes de Gahi, de Faioa et de Saint-Christophe au sud. Deux bassins occupent le coté-ouest, le bassin de Halalo au sud-ouest et la Baie de l'Ouest, au nord-ouest. Ces deux bassins communiquent avec l'océan par quatre passes ; la passe de Honikulu, au sud qui est la principale, la passe d'Avatolu, à l'ouest du bassin de Halalo, les passes de Fuga Uvea et de Fatumanini pour le bassin nord-ouest.

Le bassin sud-ouest et le bassin nord-ouest sont séparés par un compartiment surélevé, constitué par un platier récifal peu profond, limité à l’ouest par une série de hauts fonds qui le séparent de la barrière externe. La partie interne du platier est creusé par un chenal interne, dit chenal d'Utulévé, d'une longueur de $4 \mathrm{~km}$ et d'une profondeur 


\section{XIII ${ }^{\text {èmes }}$ Journées Nationales Génie Côtier - Génie Civil \\ Dunkerque, 2-4 juillet 2014}

approximative de 6 mètres (en fait une zone non hydrographiée) qui fait communiquer les bassins de l'ouest entre eux.

\section{Matériel et méthodes}

Divers instruments ont été utilisés pour l'étude hydrologique et courantologique du lagon de Wallis notamment un profileur de courant doppler (ADCP Nortek $600 \mathrm{kHz}$ ), un marégraphe houlographe enregistreur (SBE 26). Le profileur de courant ADCP Nortek a été implanté sur différents sites, du lagon et des passes, internes et externes, pour des périodes d'une durée moyenne de trois mois. Un certain nombre de stations a été positionné en arrière de la barrière récifale pour évaluer l’importance des entrées d'eau apportées par les houles océaniques. Les données sont analysées à partir des logiciels des constructeurs d'instruments et de routines Matlab afin d'établir des statistiques des processus. Notamment, une partie de l'étude des courants a porté sur l'analyse des bilans de circulation résiduelle au cours de plusieurs cycles de marée et dans différentes situations météo-océanologiques. Ces méthodes sont décrites dans HOIBIAN \& ALLENBACH, (2012).

\section{Résultats météo océanologiques}

Deux régimes météorologiques prédominent sur le site, un régime d'alizé modéré de sud-est avec des vents d'est à sud-est prépondérants pour $70 \%$ du temps et un régime de vent de nord à nord-ouest, assez forts, qui s'observe pendant $30 \%$ du temps, et correspond à ce que les wallisiens appellent le mauvais temps. (Il s'agit d'un temps variable avec des grains violents accompagnés de pluie diluvienne et des vents forts, force 7 Beaufort, dont l'apparition est +/- liée à la position de la ZCIT). Ces régimes de vents sont accompagnés le plus souvent de houles de secteurs correspondant, houles d'est à sud-est en conditions normales. Passagèrement, l'alizé peut se renforcer et donner des vents forts ( $v>8 \mathrm{~m} / \mathrm{s}$ ) accompagnés par les houles fortes correspondantes. De fortes houles de nord à nord-ouest se développent pendant les périodes de mauvais temps.

Le régime des houles a été étudié à partir des données du serveur Ifremer, Iowaga. Les statistiques des hauteurs de houles $H_{\text {sig }}$ et des périodes $T_{\text {peak }}$ sont présentées dans le tableau 1. Sur une période de temps assez longue (2005-2010), le Hsig moyen s’établit autour de 1,82 m ce qui reste modéré, La période Tpeak moyenne s'établit à 10,15 secondes. Ces houles du large ne sont pas perceptibles dans le lagon sauf conditions exceptionnelles.

La compilation des résultats de l'étude conduite depuis 2006 a permis d'apprécier différents paramètres régissant la courantologie et de quantifier les échanges entre bassins et l’océan. Ces échanges déterminent un schéma de circulation global pour le lagon de Wallis. 
Tableau 1. Caractéristiques des $H_{\text {sig }}, T_{\text {peak }}$, au point A de Wallis (Iowaga), pour la période 2005-2010.

\begin{tabular}{|c|c|c|c|c|c|c|}
\hline & \multicolumn{3}{|c|}{$\boldsymbol{H}_{\text {sig }}(\mathrm{m})$} & \multicolumn{3}{|c|}{$T_{\text {peak }}(s)$} \\
\hline & Est & Ouest & Total & Est & Ouest & Tota \\
\hline maximum & 7,55 & 4,18 & 7,55 & 21.43 & 22.25 & 22,25 \\
\hline moyenne & 1,82 & 1,83 & 1,82 & 10.95 & 9.99 & 10,15 \\
\hline
\end{tabular}

Différents types de stations sont considérés (voir tableau 2) :

- les stations d'arrière récif ; Laukahau, Tépa, Roche blanche, Malaétoli, Kanahé, ...

- les stations des passes internes ; Gahi, Vailala, Utulévé, Faioa, ...

- les stations des passes externes ; Honikulu, Avatolu, Fuga Uvea, Fatumanini.

Tableau 2. Relevés des vitesses de courant observées à différentes stations.

\begin{tabular}{lll}
\hline Stations d'arrière récif & $\operatorname{Vmax}(\mathbf{m} / \mathbf{s})$ & $\operatorname{Vmoy}(\mathbf{m} / \mathbf{s})$ \\
\hline Laukahau & 0,37 & 0,15 \\
Tepa & 0,79 & 0,40 \\
Roche blanche & 0,78 & 0,43 \\
Malaetoli & 0,26 & 0,06 \\
Kanahe & 0,26 & 0,07 \\
\hline
\end{tabular}

Stations d'arrière récif: dans l'ensemble, les stations du récif Est ont des valeurs de vitesses plus élevées que celles du récif ouest car plus exposées aux vents et houles dominants. Les directions des courants entrants sont dans le prolongement des axes des éperons-sillons de la barrière récifale, visibles sur les photographies aériennes. Quelques directions sortantes sont observées. Sur le récif ouest, les directions dominantes sont plus influencées par la bordure interne du récif.

Bilans de circulation : à titre d'exemple, nous proposons les bilans de circulation pour une station appartenant à chacun des types (voir tableaux 3, 6, 7 et 9). Les volumes sont donnés en $\mathrm{m}^{3} / \mathrm{m}^{2}$ pour un cycle de marée, pour les cellules, de surface, C1 et de miprofondeur, $C 2$ et $C 3$.

Tableau 3. Bilan de circulation à la station de Tépa pour un cycle de marée (12 HM), par différentes conditions de houle (en $\mathrm{m}^{3} / \mathrm{m}^{2}$ ) pour la cellule de surface C1 et de miprofondeur C2, C3.

\begin{tabular}{llll}
\hline Bilans station de Tepa & C1 & C2 & C3 \\
\hline Fortes houles $>\mathbf{3} \boldsymbol{m}\left(\boldsymbol{m}^{3} / \boldsymbol{m}^{2}\right)$ & 18695,54 & 10918,67 & $-134,93$ \\
Calme plat $\left(\mathbf{m}^{3} / \boldsymbol{m}^{2}\right)$ & 1068,79 & $-134,93$ & 59,93 \\
\hline
\end{tabular}




\section{XIII ${ }^{\text {èmes }}$ Journées Nationales Génie Côtier - Génie Civil \\ Dunkerque, 2-4 juillet 2014}

Le tableau 4 reprend les totaux des linéaires côtiers, déduction faite des zones exondées au droit des îlots. Le volume général pour un cycle de marée est obtenu en multipliant le linéaire de récif par le bilan en $\mathrm{m}^{3} / \mathrm{m}^{2}$. Sur la base de la station de Tépa, les volumes entrants représenteraient $573950042 \mathrm{~m}^{3}$ par cycle de marée sur $19381 \mathrm{~m}$, sur une hauteur de $1 \mathrm{~m}$. Les surélévations observées par rapport aux prévisions du SHOM, sont de l'ordre de 0,20 m pour le bassin de Mata Utu et de 0,10 m pour le bassin de Halalo, variables en fonction des conditions météo-océanologiques.

Tableau 4. Récapitulatif des linéaires de barrière récifale libre.

\begin{tabular}{llll}
\hline Secteur & Nom & Linéaire (m) & Fraction (\%) \\
\hline Nord-ouest & Baie de l'Ouest & 13701,3 & 0,25 \\
Sud-ouest & Bassin de Halalo & 14182,2 & 0,26 \\
Sud-est & Bassin de Gahi & 7910,0 & 0,14 \\
Nord-est & Bassin Est & 19380,9 & 0,35 \\
\hline & Total & $\mathbf{5 5 1 7 4 , 4}$ & $\mathbf{1 , 0 0}$ \\
\hline
\end{tabular}

Stations des chenaux internes : Quatre stations des chenaux internes ont été examinées, elles sont déterminantes dans l'établissement du schéma de circulation général (tableau 5). Deux stations appartiennent au bassin Est et jouent un rôle symétrique ; la station de la fausse passe de Vailala, au nord et la station de Gahi, au sud-est. Une station est située dans le bassin de Halalo, en bordure du chenal interne d'Utuleve qui contrôle les échanges entre les bassins sud-est et nord-ouest. La station de Faioa* a été mesurée par le SHOM et les résultats sont indiqués sur la carte des iles Wallis nº 6876.

Tableau 5. Vitesses enregistrées aux stations des chenaux internes.

\begin{tabular}{lll}
\hline Chenaux internes & $\operatorname{Vmax}(\mathrm{m} / \mathrm{s})$ & $\operatorname{Vmoy}(\mathrm{m} / \mathrm{s})$ \\
\hline Vailala & 0,61 & 0,20 \\
Gahi & 0,39 & 0,08 \\
Faioa* & 0,41 & 0,41 \\
Utuleve & 0,71 & 0,32 \\
\hline
\end{tabular}

Les deux stations placées aux extrémités du bassin de Mata’Utu montrent de grandes similitudes dans les régimes hydrologiques, avec des bilans de circulation assez largement déficitaires dans le sens de l'extérieur du bassin Est. A la fausse passe de Vailala, le courant sortant occulte le cycle de marée et domine la circulation pendant la majeure partie du temps. La majorité des écoulements est dirigée vers l'extérieur, en direction du bassin nord-ouest vers la passe de Vailala. De même, à la station de Gahi, les bilans de circulation montrent une dominance des courants sortants. 
Tableau 6. Bilans de circulation calculés à la station de Vailala.

\begin{tabular}{llll}
\hline Station de Vailala $-\mathbf{0 9 1 2}$ & $\mathbf{C 1}$ & $\mathbf{C 2}$ & $\boldsymbol{C 3}$ \\
\hline Bilan $\mathbf{V E}\left(\mathbf{m}^{3} / \mathbf{m}^{2}\right)$ & $-363,50$ & $-217,10$ & 233,00 \\
Bilan $\mathbf{M E}\left(\mathbf{m}^{3} / \mathbf{m}^{2}\right)$ & $-974,10$ & $-2342,80$ & $-3979,30$ \\
\hline
\end{tabular}

Tableau 7. Bilan calculés à la station de Gahi, pour trois cellules, de surface, C1 et de mi-profondeur, C2, C3.

\begin{tabular}{llll}
\hline Station Gahi - session 0612 & C1 & C2 & C3 \\
\hline Bilan $\mathbf{V E}\left(\mathbf{m}^{3} / \mathbf{m}^{2}\right)$ & $-1296,00$ & $-2065,30$ & $-778,60$ \\
Bilan $\boldsymbol{M E}\left(\boldsymbol{m}^{3} / \mathbf{m}^{2}\right)$ & $-1429,40$ & $-1475,20$ & $-1278,10$ \\
\hline
\end{tabular}
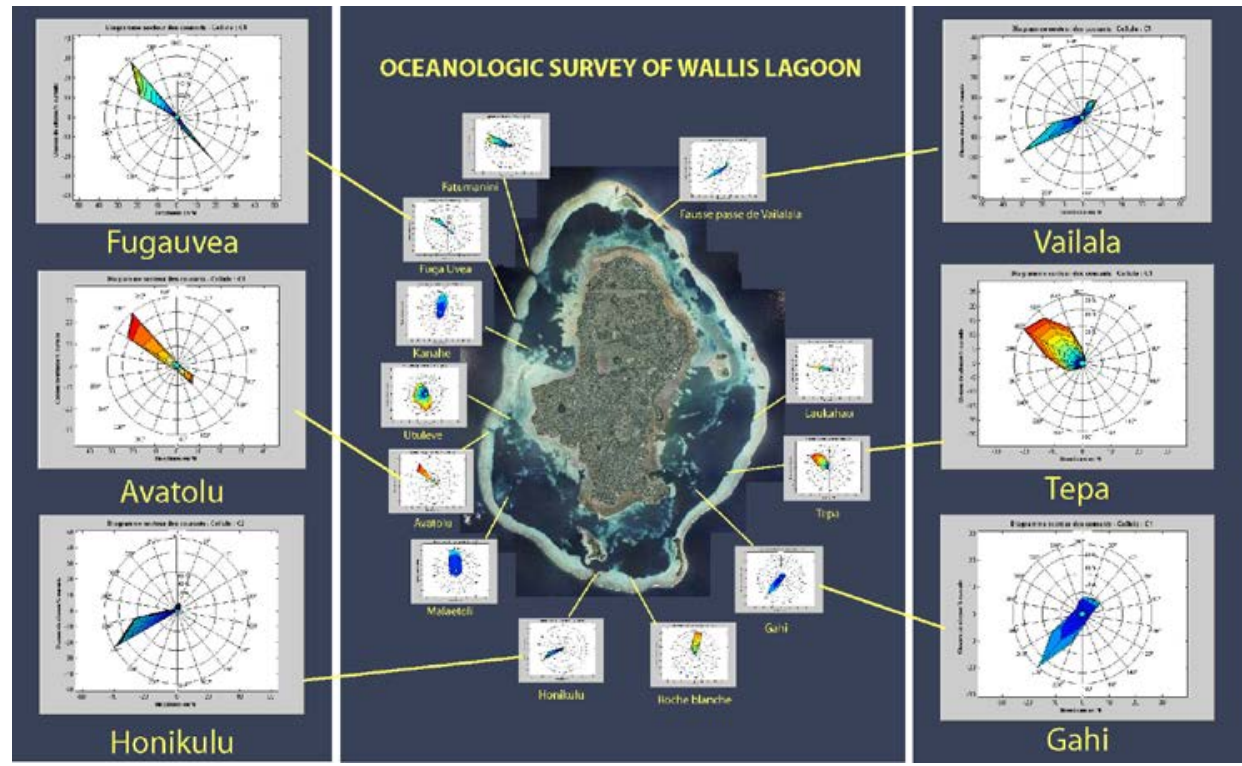

Figure 2. Carte des stations de courantologie du lagon de Wallis.

En ce qui concerne le bassin de Halalo et le chenal de Utulévé, les écoulements varient suivant le type de temps :

Par alizés de sud-est, les courants sont majoritairement dirigés vers le nord à nordouest, avec une intensité variable suivant le régime de houles et des marées.

Si les vents sont moyens à faibles, les remontées d'eau vers le nord sont modérées et il se produit des sorties d'eau assez importantes par-dessus la barrière sud-ouest. Par contre, si l'alizé est plus soutenu, la remontée vers le bassin nord-ouest est plus intense, car les sorties d'eau sont bloquées par les masses d'eau entrantes.

Par vent de nord-ouest, le tableau s'inverse totalement et les courants dominants sont dirigés vers le sud et le sud-est.

La situation des passes externes a été examinée dans un article antérieur (HOIBIAN \& ALLENBACH, 2012). Les valeurs des vitesses sont présentées dans les tableaux ciaprès. Pour résumer, le bilan de circulation général des passes externes montre un débit 


\section{XIII ${ }^{\text {èmes }}$ Journées Nationales Génie Côtier - Génie Civil \\ Dunkerque, 2-4 juillet 2014}

sortant prédominant de façon permanente. Ce n'est qu'à l'occasion des très fortes marées que les bilans arrivent à s'équilibrer. Globalement, les vitesses des courants sont largement influencées par l'amplitude des marées (tableau 8). Si celles-ci sont trop faibles (marée de mortes eaux), les courants entrants s'annulent (HOIBIAN \& ALLENBACH, 2012).

Le bilan de circulation calculé à la passe d'Avatolu (tableau 9) est assez caractéristique, avec un bilan sortant d'autant plus élevé que l’amplitude des marées est plus faible.

Tableau 8. Vitesses enregistrées dans les passes externes.

\begin{tabular}{lll}
\hline Passe externes & $\operatorname{Vmax}(\mathbf{m} / \mathbf{s})$ & $\operatorname{Vmoy}(\mathbf{m} / \mathbf{s})$ \\
\hline Honikulu & 1,72 & 0,73 \\
Avatolu & 1,60 & 0,72 \\
Fuga Uvea & 1,57 & 0,67 \\
Fatumanini & 0,71 & 0,44 \\
\hline
\end{tabular}

Tableau 9. Bilan de circulation calculé à la passe d'Avatolu pour un cycle de marée pour trois cellules de surface C1 et de mi-profondeur, C2, C3.

\begin{tabular}{llll}
\hline Passe de Avatolu - 0610 & C1 & C2 & C3 \\
\hline Bilan VE $\left(\mathbf{m}^{3} / \mathbf{m}^{2}\right)$ & $-10424,80$ & $-1734,40$ & 511,70 \\
Bilan $\mathbf{M E ~}\left(\mathbf{m}^{3} / \mathbf{m}^{2}\right)$ & $-36221,80$ & $-20657,20$ & $-16108,40$ \\
\hline
\end{tabular}

\section{Schéma de circulation global}

Les quelques points clefs présentés permettent d'esquisser un schéma de circulation en fonction du régime climatique auquel l'île est soumise. En ce qui concerne les conditions standard (alizé de sud-est), deux sous-régimes sont distingués :

- En régime calme:

Dans ce cas, le plus fréquent, les houles sont modérées et les entrées d'eau aussi ;

Dans le bassin de Mata'Utu, les eaux s'écoulent, vers le sud, en direction de la passe de Honikulu et le bassin de Halalo, via la passe de Gahi, et vers le nord, en direction du bassin nord-ouest, par la fausse passe de Vailala, (observations).

Dans le bassin de Halalo, du fait du régime de houles modéré, l'eau s'évacue pardessus la barrière récifale surbaissée par rapport à la barrière au vent (à l’Est).

- En régime d'alizé renforcé :

Les houles sont plus fortes et les entrées d'eau aussi. Les eaux s’écoulent du bassin Est comme dans le cas précédent. La différence intervient au niveau du bassin de Halalo.

Dans le bassin de Halalo, du fait de la réfraction des houles autour de la pointe sud de l'île, il se produit aussi des entrées d'eau sur la barrière récifale. De ce fait, les masses d'eau en provenance du bassin de Mata Utu ne peuvent s'évacuer par-dessus la barrière et sont rejetées dans le bassin nord-ouest par chenal interne d’Utulévé. 
Par mauvais temps de nord-ouest, un seul régime de fortes houles est pris en compte. Dans ces conditions, les masses d'eaux qui entrent par le bassin nord-ouest ne peuvent s'évacuer totalement par les deux passes de Fatumanini et de Fuga Uvea et sont refoulées dans les bassins lagonaires adjacents, principalement par le chenal interne d'Utulévé et par la fausse passe de Vailala. Les eaux s'évacuent par la passe d'Avatolu, par la passe d'Honikulu et par-dessus la barrière jusque dans le bassin de Mata’Utu, où elles remontent exceptionnellement.

\section{Conclusions}

Quelques observations de sites clef, permettent d'esquisser un schéma de circulation général qui distingue deux régimes principaux ; le régime d'alizé de sud-est et le régime de nord-ouest. Le mécanisme reste identique dans les deux cas; Les entrées d'eau causées par le déferlement des houles sur la barrière récifale conduisent à une surélévation du plan d'eau du bassin considéré. Ceci crée un gradient de pression qui conduit à l'évacuation des eaux excédentaires, via les passes internes, vers les bassins situés à l'opposé de l'île, où elles s’évacuent par les passes et/ou par-dessus la barrière.
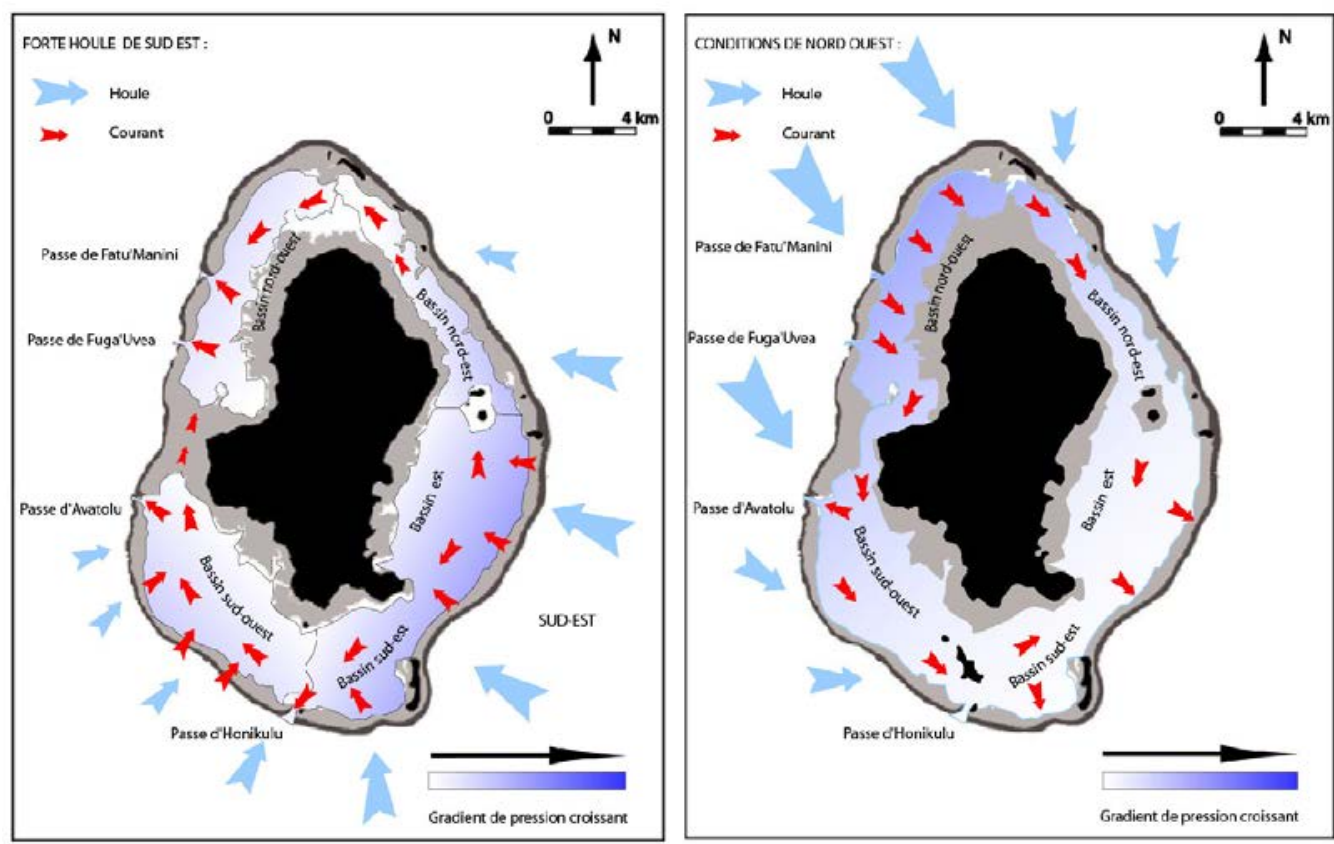

Figure 3. Schéma de circulation par différentes conditions météorologiques.

\section{Références bibliographiques}

HOIBIAN T., ALLENBACH M. (2012). Caractérisation du bilan de circulation hydrologique du lagon de Wallis (Pacifique sud-ouest). XII ${ }^{\text {èmes }}$ Journées Nationales Génie Côtier - Génie Civil, Cherbourg, pp 933-940. http://dx.doi.org/10.5150/jngcgc.2012.102-H 